\title{
Assembling Similar Tracking Approaches in Order to Strengthen Performance
}

\author{
Edgar Reyna-Ayala, Santiago E. Conant-Pablos, and Hugo Terashima-Marín \\ Tecnológico de Monterrey \\ Av. Eugenio Garza Sada 2051, Monterrey, N.L. \\ 64849 Mexico \\ $\{$ a00800773, sconant, terashima\}@itesm.mx
}

\begin{abstract}
In this paper we present a novel ensemble of two similar tracking approaches, which independently present good performance for different video sequences. We propose that by combining the response of these tracking approaches, we can strengthen their detecting capability and therefore increase the tracking performance of the ensemble. The Tracking-Learning-Detection (TLD) and the LocalTLD are the approaches we chose for building our ensemble. Our main motivation for assembling these two approaches is that both approaches focus on particular instances of an object and also manage different object representation, for instance, the TLD works reasonably well for planar rigid objects due to the global classifier it includes, meanwhile the LocalTLD focuses on invariant local features and is able to overcome the planar assumption. Combining these approaches, we are able to take advantage of their best qualities and overcome their biggest problems. For introducing our method, we first need to review the principal components of the two chosen approaches, and then we finally introduce the ensemble. The proposed ensemble is compared against results of the independent approaches using a data set of 10 video sequences, showing, in general, a significant improvement.
\end{abstract}

Keywords: Tracking, Semi-supervised Learning, Online Learning, Random Ferns, Template-based Classifier.

\section{Introduction}

The general case of tracking arbitrary objects in unconstrained environments is very challenging due to the existence of several factors that act as distractors such as changes in appearance, varying lighting conditions, cluttered background, the emergence of regions having appearance similar to the target. In some applications, the object to be tracked is known in advance and it is possible to incorporate specific prior knowledge. However, tracking arbitrary objects by simply specifying a single training example is a challenging open problem that deserves particular attention. In this scenario, the tracker must be able to model the object by generating and labeling image features and learning different appearances of the object. This basic formulation naturally leads to the semi-supervised learning approach. 
Tracking by detection is a tracking technique that recent works [1], [2], 3] have shown it provides promising results. These methods train a discriminative classifier in an online fashion to separate the object from the background. These classifiers bootstrap themselves by using the current tracker state to extract positive and negative examples from the current frame.

The framework proposed in this investigation is built upon two approaches that have previously presented good results, and since both are structured in a similar fashion, they can be easily adapted for working together. The proposed ensemble is compared against results of the independent approaches using a data set of video sequences, showing, in general, a significant improvement. For introducing our method, we first review similar approaches in the state of the art, this is presented in Section 2, then we review the principal components and explain the ensemble in Section 3, in Section 4 we present the results obtained with the framework. Conclusions are finally given in Section 5 .

\section{Related Work}

Most of the methods on the state of the art focus their efforts to solve the problem of tracking arbitrary objects in unconstrained environments by using different techniques like local feature matching, ensemble classifiers, keypoint classification, specialized filters, etc. A framework for adaptive visual object tracking based on structured output prediction is presented in 3. By explicitly allowing the output space to express the needs of the tracker, they are able to avoid the need for an intermediate classification step. This method uses a kernelized structured output support vector machine (SVM), which is learned online to provide adaptive tracking. To allow for real-time application, they introduce a budgeting mechanism that prevents the unbounded growth in the number of support vectors, which would otherwise occur during tracking.

In [4] a tracking method based on the TLD system [5] is presented that exploits the context of the object of interest by characterizing it in two terms: Distractors and Supporters. Distractors are defined as regions that have similar appearance as the target and consistently co-occur. They keep track of these distractors to avoid drifting. Supporters are defined as local features around the target with consistent co-occurrence and motion correlation. Their main purpose is to make a stronger validation that the object is being tracked correctly.

The ALIEN algorithm [6] is based on local features for building the object model and a context model, which represents the object surrounding. Pernici et. al [6] propose a technique mainly motivated by Scale Invariant Features Transformation (SIFT features) [7] and by the underlying image formation process. It comprises multiple instances of local features combined with a global shape prior, expressed in terms of a 2D similarity transformation. This novel representation is exploited in a discriminative background/foreground online tracking (by detection) method, which performs feature selection and feature update. The resulting technique allows tracking to continue under severe visibility artifacts. 


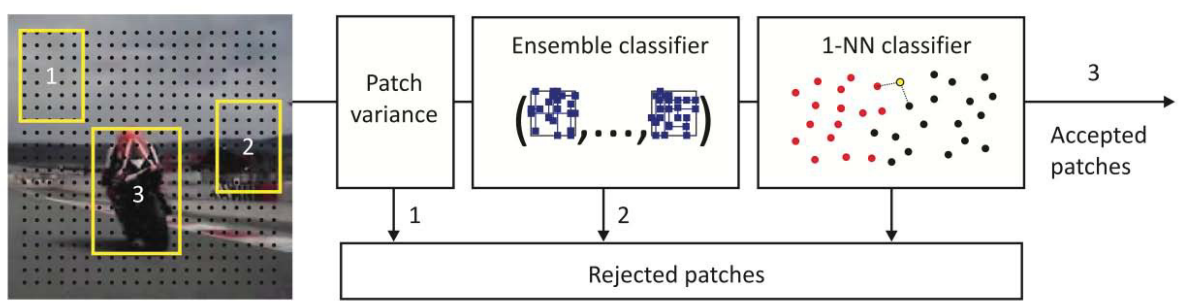

Fig. 1. Block diagram of the TLD detecting component, from $[5]$

\section{Implementation}

The framework proposed in this investigation is built upon two approaches that have previously presented good results when compared with the state of the art. The TLD [5] and the LocalTLD [8] are the approaches we chose for building our framework, since they are structured in a similar fashion, they can be easily adapted for working together. Our main motivation for assembling these two approaches is that both approaches focus on particular instances of an object and also manage different object representation, for instance, the TLD framework works reasonably well for planar rigid objects due to the global classifier it includes, meanwhile the LocalTLD framework focuses on invariant local features and is able to overcome the planar assumption. Therefore, we propose that by combining the responses of these two approaches, we can strengthen their detecting capabilities and hence increase the tracking performance of the ensemble. For introducing our method, we first need to review the principal components of the TLD and the LocalTLD, and then we finally introduce the ensemble.

\subsection{Tracking-Learning-Detecting}

The TLD system [5] retakes Viola-Jones cascade methodology [9] to design a global object detector. A new paradigm for learning from structured unlabeled data called P-N Learning is introduced, where the structure in the data is exploited by so called positive and negative structural constraints, which enforces certain labeling of the unlabeled set. The TLD system divides the process of tracking an unknown object into three basic tasks: tracking, learning and detecting. The tracking component and the detecting component represent different estimations of the object.

The Tracking Component. The tracking component estimates the motion of the object between two consecutives frames, and therefore can discover non-learned object appearances to generate training examples for the detecting component. This statement establishes the tracking component as the main component for triggering the learning process. The tracking component is based on Median-Flow tracker extended with failure detection [10]. 
The Detecting Component. The detecting component scans the whole frame in order to localize the object based on the object appearances previously observed and learned. Once the object is located, it corrects the tracking component response. For this component, Kalal et. al [5] retakes Viola-Jones cascade methodology 9 to design a global object detector. The cascade methodology is divided into three stages, as shown in Figure 1 a variance filter, an ensemble classifier and a template-based classifier. This methodology can be viewed as an object specific focus-of-attention mechanism, which provides statistical guarantees that discarded regions are unlikely to contain the object of interest [9].

The Learning Component. The learning component estimates the detecting component error and trains it to avoid making the same mistakes in the future. The learning component uses the methodology known as P-N learning [5]. The key idea of P-N learning is that two types of experts can identify the detector errors. The P-expert assumes that the object moves along a trajectory and extracts examples from such trajectory and the $\mathrm{N}$-expert assumes that the object can occupy only a single location and extracts examples from the annular area of the trajectory, so they both depend on the validation of the trajectory given by the tracking component. Both experts may produce errors themselves; however, their independence enables mutual compensation of their errors.

\subsection{LocalTLD}

This approach is embedded into the Tracking-Learning-Detection (TLD) framework by performing a set of changes in the detection stage. In [8] the authors propose using invariant local features and a global appearance validation for assisting a robust object tracker initialized by a single example. The authors show how measuring the density of positive local features given by a binary classifier is a good signal of the objects presence, and in combination with a global appearance validation it yields a strong object detector.

The Tracking Component. LocalTLD also uses the Median-Flow tracker extended with failure detection, as in [10]. The original implementation initializes an uniform $10 \times 10$ keypoints grid within the last known bounding box and track those points. In the variant approach, a slightly modification is made on the points to track, by randomly select 100 keypoints given by the feature extraction stage since they are more likely to be stable for tracking purposes.

The Detecting Component. For this component the author uses three stages for the cascade methodology [9, as shown in Figure 2, an ensemble classifier for classifying the local features as belonging to the object or the background, then a feature density filter acting as the object specific focus-of-attention mechanism and finally, a template-based classifier similar to the one implemented in the TLD framework. 


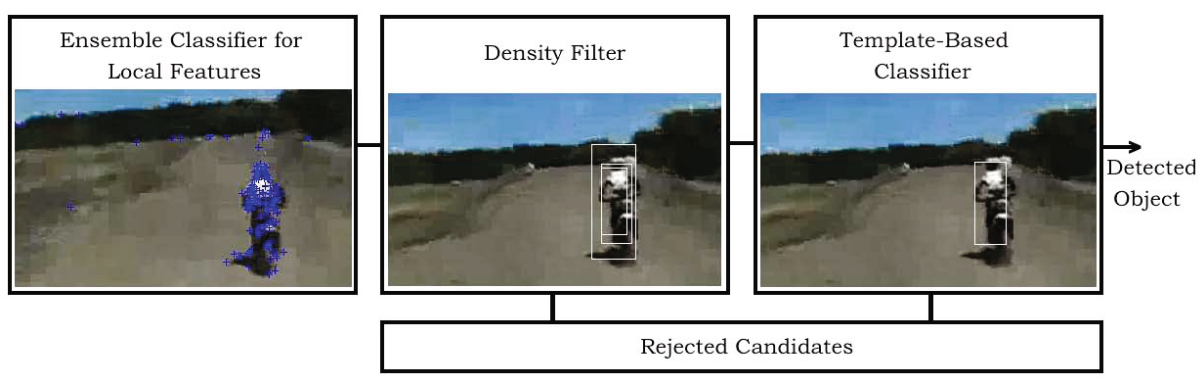

Fig. 2. Local features are extracted from the image and classified by an ensemble classifier as either belonging to the object (crosses) or the background. Then the feature density filter acts as an object specific focus-of-attention mechanism. Finally each candidate is passed through the global validation stage given by a template-based classifier.

The Learning Component. The learning component uses the methodology known as P-N learning [5], as in the TLD framework.

\subsection{Assembling Approaches}

After a careful analysis of the two approaches described before, we can conclude that in many cases there might be circumstances in which one approach is not suitable enough; in other words, none of the approaches can guarantee a competent performance for all kind of circumstances. We can observe in the behavior comparison of the two approaches (see Figure 3), that in certain circumstances the TLD outperforms the LocalTLD and in other distinct circumstances the LocalTLD outperforms the TLD.

By assembling these two similar approaches we provide the framework with new resources in such a way that when one detector is not able to detect the object, the second detector is most probably able to detect it, providing not only a valid response but also new information to update both detectors.

The Tracking Component. Since both approaches are based on the MedianFlow tracker [10, we implement it as well.

The Detecting Component. In this component, we implement both cascade detectors independently, as shown in Figure 4 , each detector builds its own object model, has its own database of examples and computes its own posterior probabilities for the ensemble classifier. Although there are many ways to combine the response of various detectors, we keep them both working simultaneously and average their responses in order to retrieve a definitive response for the detecting stage.

For averaging the responses of the detectors, it is necessary to take into account two metrics, an overlapping measure with the tracking component response 


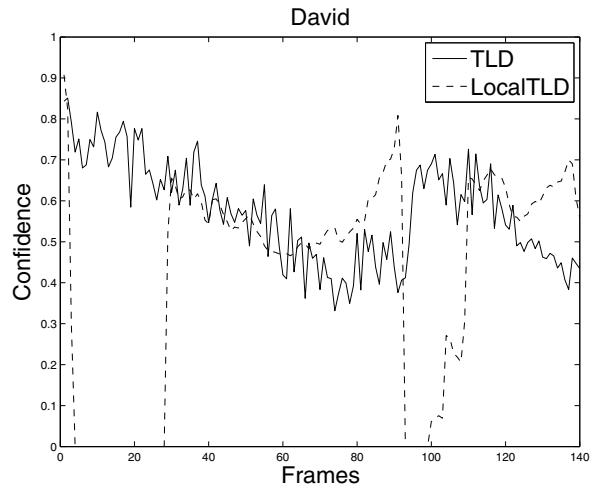

(a) David

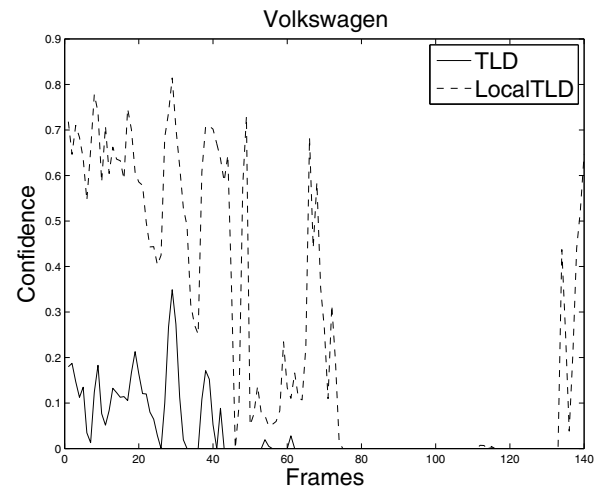

(b) Volkswagen

Fig. 3. Comparison of performance between TLD and LocalTLD. In this graphs we can observe the average performance of both approaches in the same video sequences. We can observe how in the David sequence the TLD framework overcomes the LocalTLD, but in the Volkswagen sequence occurs the other way around.

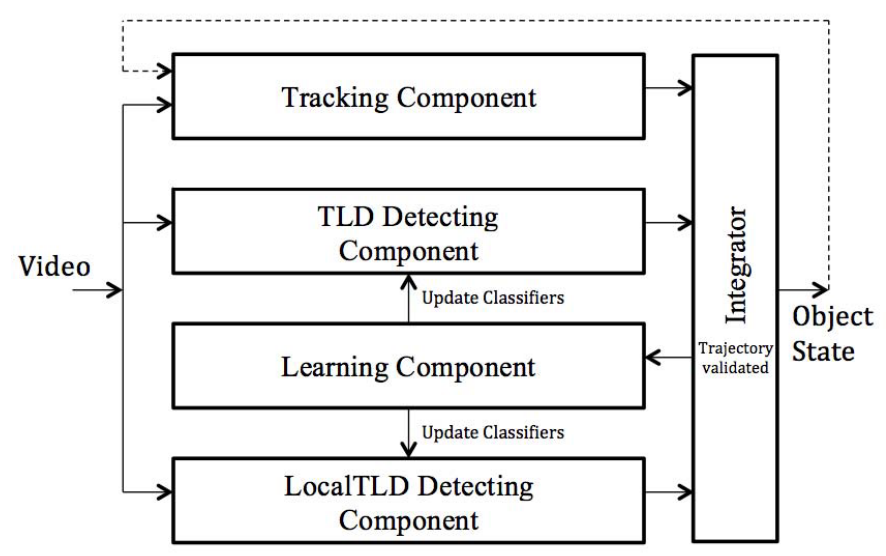

Fig. 4. Overview of the Ensemble analytical model

and a similarity measure computed as described in [5]. Then we proceed as follow, if both detectors have a high overlapping measure (above a predefined threshold), which means that both detectors agree with the location of the tracking component response, then we average the three responses. Moreover, if only one detector has a high overlapping measure, it means that the second detector does not agree with the location of the tracking component response. In this case, in order to determine which detector is correct, we have to compute the similarity of the response of each detector, and then the response with higher similarity is considered correct. If the selected response is also the one with high overlapping measure, then the response of the other detector is discarded and only average 
the selected response with the traking component response, otherwise the tracking component is reinitialized with the location of the selected response. Finally, if none of the detectors has a high overlapping measure, it means that no detector agrees with the location of the tracking component response, and we have to determine which response is correct similarly as described before. This methodology is effective for reinitializing the tracking component when it has lost the object and avoids introducing inappropriate information to the databases.

The Learning Component. The P-N learning method is implemented for the learning component of both detectors. As mentioned before, the validation of the trajectory given by the tracking component triggers the learning process. In order to validate the trajectory we need to compute the similarity of the tracking component response as in [5]. If the similarity is higher than a threshold then it is validated, otherwise it is not validated and the learning process is not executed. The learning process is an important process in the ensemble and its absence can lead to an inevitable loss of important information and in extreme cases the complete loss of the actual appearance of the object.

In the individual implementation of each detector, this dependency on the tracking component represents a problem, because there might be circumstances in which the detector is not competent enough, and therefore the similarity of the tracking component response will be mostly low, even though the trajectory is correct.

In our implementation, we propose to ease the restriction of the validation of the trajectory by exploiting the two detectors. We state that the validation of the trajectory by, at least, one of the detectors is reliable enough for triggering the learning process in both detectors. This modification causes that the databases of the detectors are no longer entirely independent, but also provokes that the detectors no longer lose valuable information in circumstances in which they cannot properly detect the object. The good performance in the longer video sequences, presented in Section 4, is in part because this partial independence between the detectors.

\section{Experiments}

For the experiments, we tested the proposed ensemble with the publicly available TLD data set, introduced in [5] and consisting of 10 video sequences. To compare our results against those of TLD and LocalTLD published in [8], we use metrics for precision, recall and F-measure. The sequences contain various objects in challenging conditions that include abrupt camera motion, motion blur, appearance change and partial or complete occlusions. Table 1 lists the properties of the sequences.

For the evaluation method, every box that has an overlapping percentage with the ground truth higher than $50 \%$ is considered as a valid prediction of the object location. The precision metric is computed as the number of valid predictions divided by the total number of predictions. The recall metric is computed as the 
Table 1. TLD data set description, from [5]

\begin{tabular}{|l|c|c|c|c|c|c|c|c|}
\hline Sequence & Frames & $\begin{array}{c}\text { Mov. } \\
\text { camera }\end{array}$ & $\begin{array}{c}\text { Partial } \\
\text { occ. }\end{array}$ & $\begin{array}{c}\text { Complete } \\
\text { occ. }\end{array}$ & $\begin{array}{c}\text { Pose } \\
\text { change }\end{array}$ & $\begin{array}{c}\text { Illum. } \\
\text { change }\end{array}$ & $\begin{array}{c}\text { Scale } \\
\text { change }\end{array}$ & $\begin{array}{c}\text { Similar } \\
\text { objects }\end{array}$ \\
\hline \hline 1. David & 761 & yes & yes & no & yes & yes & yes & no \\
2. Jumping & 313 & yes & no & no & no & no & no & no \\
3. Pedestrian 1 & 140 & yes & no & no & no & no & no & no \\
4. Pedestrian 2 & 338 & yes & yes & yes & no & no & no & yes \\
5. Pedestrian 3 & 184 & yes & yes & yes & no & no & no & yes \\
6. Car & 945 & yes & yes & yes & no & no & no & yes \\
7. Motocross & 2665 & yes & yes & yes & yes & yes & yes & yes \\
8. Volkswagen & 8576 & yes & yes & yes & yes & yes & yes & yes \\
9. Car Chase & 9928 & yes & yes & yes & yes & yes & yes & yes \\
10. Panda & 3000 & yes & yes & yes & yes & yes & yes & no \\
\hline
\end{tabular}

Table 2. Fixed Parameters

\begin{tabular}{|c|c|}
\hline Parameter & Value \\
\hline \hline Minimum window size & 15 pixels \\
\hline Number of fern structures for TLD detector & 10 structures \\
\hline Number of fern structures for LocalTLD detector & 10 structures \\
\hline Size of every fern structure for TLD detector & 13 comparisons \\
\hline Size of every fern structure for LocalTLD detector & 14 comparisons \\
\hline Template-based classifier patch size & 15 pixels \\
\hline Maximum number of Template-based classifier examples & 100 examples \\
\hline
\end{tabular}

number of valid predictions divided by the number of actual ocurrences of the object, given by the ground truth. Finally, the F-measure is the harmonic mean of the two measures described above.

\subsection{Parameters}

The classifiers have certain parameters that determine their accuracy and speed, such as the minimum window size, the number of fern structures, the size of the fern structures, and the patch size, among others. For each one of the approaches, in the original implementations, the authors fix these parameters according to the experiments described in [5], 8] and claim that the choice of the parameters is not critical. For the sake of consistency, we use the same parameter values. In Table 2 we show the fixed value for each parameter. The minimum window size is the minimum value that will be acceptable for any candidate bounding box dimensions. For the ensemble classifier there are two main parameters the number of fern structures that contain the ensemble and the size of every fern structure. For the template-based classifier there are two main parameters as well, the patch size of the examples in the database and the maximum number of examples that can be stored in the database at the time.

\subsection{Results}

In order to validate our hypothesis we compare the performance of the approaches with the publicly available TLD data set, consisting of 10 video sequences, and compare our measure of precision, recall and F-measure with the 
Table 3. Tracking Performance Analisys

\begin{tabular}{|l|c|c|c|c|c|c|c|c|c|c|}
\cline { 3 - 11 } \multicolumn{1}{c|}{} & \multicolumn{3}{c|}{ TLD } & \multicolumn{3}{c|}{ LocalTLD } & \multicolumn{3}{|c|}{ Ensemble } \\
\hline Sequence & Frames & $\mathrm{P}$ & $\mathrm{R}$ & $\mathrm{F}$ & $\mathrm{P}$ & $\mathrm{R}$ & $\mathrm{F}$ & $\mathrm{P}$ & $\mathrm{R}$ & $\mathrm{F}$ \\
\hline 1. David & 761 & $\mathbf{1 . 0 0}$ & $\mathbf{1 . 0 0}$ & $\mathbf{1 . 0 0}$ & 0.92 & 0.91 & 0.91 & $\mathbf{1 . 0 0}$ & $\mathbf{1 . 0 0}$ & $\mathbf{1 . 0 0}$ \\
2. Jumping & 313 & $\mathbf{0 . 9 9}$ & $\mathbf{0 . 9 9}$ & $\mathbf{0 . 9 9}$ & 0.66 & 0.55 & 0.6 & 0.92 & 0.83 & 0.87 \\
3. Pedestrian 1 & 140 & $\mathbf{1 . 0 0}$ & $\mathbf{1 . 0 0}$ & $\mathbf{1 . 0 0}$ & 0.18 & 0.17 & 0.17 & 0.66 & 0.39 & 0.49 \\
4. Pedestrian 2 & 338 & 0.89 & 0.92 & 0.91 & $\mathbf{0 . 9 6}$ & $\mathbf{0 . 9 3}$ & $\mathbf{0 . 9 5}$ & 0.74 & 0.86 & 0.79 \\
5. Pedestrian 3 & 184 & $\mathbf{0 . 9 9}$ & $\mathbf{1 . 0 0}$ & $\mathbf{0 . 9 9}$ & 0.83 & 0.94 & 0.88 & 0.75 & 0.87 & 0.80 \\
6. Car & 945 & 0.92 & 0.97 & 0.94 & $\mathbf{0 . 9 5}$ & 0.97 & $\mathbf{0 . 9 6}$ & 0.94 & $\mathbf{0 . 9 9}$ & $\mathbf{0 . 9 6}$ \\
7. Motocross & 2665 & 0.67 & 0.58 & 0.62 & 0.70 & $\mathbf{0 . 6 4}$ & $\mathbf{0 . 6 7}$ & $\mathbf{0 . 7 1}$ & $\mathbf{0 . 6 4}$ & $\mathbf{0 . 6 7}$ \\
8. Volkswagen & 8576 & 0.54 & 0.4 & 0.45 & $\mathbf{0 . 7 1}$ & 0.88 & $\mathbf{0 . 7 8}$ & 0.65 & $\mathbf{0 . 9 6}$ & $\mathbf{0 . 7 8}$ \\
9. Car Chase & 9928 & 0.5 & 0.4 & 0.45 & 0.71 & 0.34 & 0.46 & $\mathbf{0 . 6 9}$ & $\mathbf{0 . 4 5}$ & $\mathbf{0 . 5 5}$ \\
10. Panda & 3000 & 0.32 & 0.34 & 0.33 & 0.35 & 0.21 & 0.26 & $\mathbf{0 . 7 7}$ & $\mathbf{0 . 6 5}$ & $\mathbf{0 . 7 0}$ \\
\hline Average & $\mathbf{2 6 8 5 0}$ & 0.56 & 0.47 & 0.5 & 0.68 & 0.58 & 0.6 & $\mathbf{0 . 7 1}$ & $\mathbf{0 . 7}$ & $\mathbf{0 . 6 8}$ \\
\hline
\end{tabular}

results published in [8]. The results of our experiments are shown in Table 3 We can observe in bold font the higher measures for each video sequence; in average the ensemble outperforms the TLD and the LocalTLD in the tracking tasks. Also we can observe an improved performance in comparison to the results obtained by TLD and Local TLD in the longer video sequences (over 2000 frames), with this we can validate our initial assumption that by combining the two approaches, we can increase the tracking performance of the ensemble. The framework was tested at an average frame rate of $7 \mathrm{fps}$ on a MacBook running OSX Mavericks with a $2.9 \mathrm{GHz}$ Intel Core 17.

It is important to point out that, on one hand the performance shown by the ensemble in very long video sequences overcomes the two approaches in which it is embedded, but on the other hand, in short video sequences the ensemble performance may slightly decrease, this is because the template-based classifiers are not entirely independent from each other, as explained in section 3.3. However further research about this point is considered as future work.

\section{Conclusion}

In this paper we address the problem of tracking an unknown object in long video sequences under complex interactions, such as changes in appearance, varying lighting conditions, cluttered background, etc. We introduce a novel ensemble of two similar tracking approaches, which each one of them presents good performance. We validate the assumption that by combining the responses of the two approaches, we can strengthen their detecting capabilities and therefore increase the tracking performanceof the ensemble. We chose the TLD and LocalTLD approaches among others because they are similarly structured and manage similar resources; also both approaches focus on particular instances of the object, manage different object representation and fit perfectly for the ensemble purposes.

We consider the parallel training of both detectors by one single validation of the trajectory as one of the principal characteristics of the ensemble, however the correlation between the classifiers is a characteristic that must be suppressed, further research about this subject is considered future work. Also as future work 
we are contemplating the possibility of adding another specialized detector to improve performance in circumstances in which none of the included approaches performs properly. Furthermore, it would be interesting to modify the scheme by which both detectors interact, for instance, introduce some intelligent method to determine which detector is most suitable for detecting according to the evolution of the video sequence and the circumstances in it.

Acknowledgment. This research effort was supported by the strategic project on Pattern Recognition and its application in Optimization and Medicine (grant PRY075) funded by Tecnolgico de Monterrey, and the National Council for Science and Technology (CONACyT).

\section{References}

1. Avidan, S.: Ensemble tracking. IEEE Transactions on Pattern Analysis and Machine Intelligence 29(2), 261-271 (2007)

2. Babenko, B., Yang, M.H., Belongie, S.: Visual tracking with online multiple instance learning. In: IEEE Conference on Computer Vision and Pattern Recognition, CVPR 2009, pp. 983-990 (2009)

3. Hare, S., Saffari, A., Torr, P.H.S.: Struck: Structured output tracking with kernels. In: 2011 IEEE International Conference on Computer Vision (ICCV), pp. 263-270 (2011)

4. Dinh, T.B., Vo, N., Medioni, G.: Context tracker: Exploring supporters and distracters in unconstrained environments. In: 2011 IEEE Conference on Computer Vision and Pattern Recognition (CVPR), pp. 1177-1184 (2011)

5. Kalal, Z., Mikolajczyk, K., Matas, J.: Tracking-learning-detection. IEEE Transactions on Pattern Analysis and Machine Intelligence 34(7), 1409-1422 (2012)

6. Pernici, F., Del Bimbo, A.: Object tracking by oversampling local features. IEEE Transactions on Pattern Analysis and Machine Intelligence (TPAMI) (in press, 2014)

7. Lowe, D.G.: Distinctive image feature from scale-invariant keypoints. International Journal of computer vision, 91-110 (Novemberl 2004)

8. Torres-Nogales, A., Conant-Pablos, S., Terashima-Marín, H.: Local features classification for adaptive tracking. Mexican International Conference on Artificial Intelligence (2012)

9. Viola, P., Jones, M.: Rapid object detection using a boosted cascade of simple features. In: Proceedings of the 2001 IEEE Computer Society Conference on Computer Vision and Pattern Recognition, CVPR 2001, vol. 1, pp. I-511 - I-518 (2001)

10. Kalal, Z., Mikolajczyk, K., Matas, J.: Forward-backward error: Automatic detection of tracking failures. In: 2010 20th International Conference on Pattern Recognition (ICPR), pp. 2756-2759 (August 2010) 